\title{
Persistent Left Superior Vena Cava in Cardiac Congenital Surgery
}

\author{
Cristina Giuliani-Poncini • Marie-Hélène Perez • \\ Jacques Cotting • Michel Hurni • Nicole Sekarski • \\ Jean-Pierre Pfammatter $\cdot$ Stefano Di Bernardo
}

Received: 11 March 2013/Accepted: 18 June 2013/Published online: 3 July 2013

(C) Springer Science+Business Media New York 2013

\begin{abstract}
Persistent left superior vena cava (LSVC) is a relatively frequent finding in congenital cardiac malformation. The scope of the study was to analyze the timing of diagnosis of persistent LSVC, the timing of diagnosis of associated anomalies of the coronary sinus, and the global impact on morbidity and mortality of persistent LSVC in children with congenital heart disease after cardiac surgery. Retrospective analysis of a cohort of children after cardiac surgery on bypass for congenital heart disease. Three hundred seventy-one patients were included in the study, and their median age was 2.75 years (IQR 0.65-6.63). Forty-seven children had persistent LSVC $(12.7 \%)$, and persistent LSVC was identified on echocardiography before surgery in 39 patients $(83 \%)$. In three patients (6.4\%) with persistent LSVC, significant inflow obstruction of the left ventricle developed after surgery leading to low output syndrome or secondary pulmonary hypertension. In eight patients (17\%), persistent LSVC was associated with a partially or completely unroofed coronary
\end{abstract}

C. Giuliani-Poncini · M.-H. Perez $\cdot$ J. Cotting Pediatric Intensive Care Unit, University Hospital Center and University of Lausanne, Lausanne, Switzerland

\section{Hurni}

Cardiovascular Surgery Department, University Hospital Center and University of Lausanne, Lausanne, Switzerland

N. Sekarski · S. Di Bernardo ( $₫)$

Pediatric Cardiology Unit, University Hospital Center and

University of Lausanne, Lausanne, Switzerland

e-mail: stefano.di-bernardo@chuv.ch

J.-P. Pfammatter

Pediatric Cardiology Unit, University Hospital of Bern, Bern, Switzerland sinus and in two cases (4\%) with coronary sinus ostial atresia. Duration of mechanical ventilation was significantly shorter in the control group (1.2 vs. 3.0 days, $p=0.04$ ), whereas length of stay in intensive care did not differ. Mortality was also significantly lower in the control group ( 2.5 vs. $10.6 \%, p=0.004)$. The results of study show that persistent LSVC in association with congenital cardiac malformation increases the risk of mortality in children with cardiac surgery on cardiopulmonary bypass. Recognition of a persistent LSVC and its associated anomalies is mandatory to avoid complications during or after cardiac surgery.

Keywords Congenital heart disease - Cardiopulmonary bypass Pediatric $\cdot$ Persistent left superior vena cava

\section{Introduction}

Persistent left superior vena cava (LSVC) is the most common anomaly among variations of thoracic venous system $[6,8,13,14]$. This anomaly is thought to be due to the persistence of the left anterior cardinal vein, which normally involutes to give the ligament of Marshall or the ligament of the LSVC $[6,13]$.The development of major systemic veins is a complex process resulting from the growth and involution of different venous drainage systems during gestation $[13,14]$. The incidence of persistent LSVC is $0.3-0.5 \%$ in the general population and between 3 and $10 \%$ in patients with congenital heart disease. Persistent LSVC is most frequently associated with atrial septal defects (ASDs), coarctation of the aorta, transposition of the great arteries, mitral valve atresia, and tetralogy of Fallot, but it can be found in complex congenital malformations, such as single ventricle $[4,6,8,13,14]$. 
The majority of persistent LSVC drains into the coronary sinus, and in $92 \%$ of cases the venous blood reaches the right atrium without clinical consequences. Most of the time, the right superior vena cava persists, and only 10 and $20 \%$ of cases of persistent LSVC are associated with absent right superior vena cava. The connection between the two venae cavae, i.e., the innominate vein, may be present or completely atretic, thus implying that the left and right venous drainages are no longer connected. In the remaining cases $(8 \%)$, the persistent LSVC may drain directly into the left atria or into an abnormal coronary sinus with an opened roof into the left atria. Patients may present with cyanosis, polycythemia, and increased risk of systemic embolism [5, 6, 17].

Reports in the literature concerning persistent LSVC are centered on difficulties, in patient without congenital heart disease, in placing central venous catheter or pacemaker lead insertions or related complications [12, 15, 17, 22]. In contrast, there are many reports of persistent LSVC associated with rare combination of congenital heart disease or reports on persistent LSVC and associated anomalies of the coronary sinus $[2,3,21]$. The global impact of persistent LSVC has, to our knowledge, never been evaluated during the perioperative and postoperative period of cardiac surgery for congenital heart disease in children.

We designed this study to analyze the timing of diagnosis of the persistent LSVC, the timing of diagnosis of associated anomalies of the coronary sinus, and the global impact on morbidity and mortality of persistent LSVC in children with congenital heart disease after cardiac surgery.

\section{Materials and Methods}

\section{Ethics}

Our institutional Research Ethics Committee approved the study and specifically waived the requirement for informed consent.

\section{Study Design}

This study was a retrospective analysis of a cohort of children admitted in the PICU after cardiac surgery for congenital heart disease on bypass in Bern and Lausanne, Switzerland.

\section{Study Population}

The study population comprised children (age 0-16 years) who were admitted to our PICU after cardiac surgery for congenital heart disease.
Data

Data collected included charts and echocardiography report review. Data after surgery were prospectively collected using our point-of-care Clinical Information System (MetaVision and MVcentral; iMDsoft, Needham, Massachusetts, USA); and retrospectively analyzed.

\section{Outcome Measures}

Outcome measures included timing of diagnosis of persistent LSVC (before, at, or after surgery) and timing of diagnosis of associated anomalies of the coronary sinus (before, at, or after surgery). Postsurgical outcomes were compared between children with persistent LSVC and a control group. Mortality, Pediatric Intensive Care Unit (PICU) length of stay (LOS) and duration of mechanical ventilation (MV) were the main outcome measures.

\section{Definitions}

Definitions used in the study are as follows.

\section{PRISM}

The Paediatric Risk of Mortality score was developed from the physiologic stability index to decrease the number of physiologic variables required for PICU mortality risk assessment and to obtain an objective weighting of the remaining variables [11].

\section{RACHS}

The Risk Adjusted Classification for Congenital Heart Surgery was created to compare in-hospital mortality for groups of children undergoing surgery for congenital heart disease [9]. RACHS stratifies anatomic diversity into six categories based on age, type of surgery performed, and similar in-hospital mortality [18]. Patients with RACHS 1 and RACHS 6 categories were excluded because of the lack of patient with persistent LSVC in both groups and those of RACHS 5 because there were few patients in this category.

Statistical Analysis

Data are presented as median and interquartile range. Odds ratios (ORs), followed by their $95 \%$ confidence intervals (CIs) in parentheses, were used to estimate strength of association. Chi square or Fisher's exact tests were used to assess the association between persistent LSVC and mortality, LOS, or MV. Spearman's and Wilcoxon's rank sum test were used to test associations for skewed variables. To estimate the effect of exposure (persistent LSVC) on 
mortality, LOS, or MV, we used univariate logistic regression. For statistical analysis, we used Stata 10.1 version, and the significance level was set at $5 \%$.

\section{Results}

Demographics

A total of 371 patients were included in the study. Age ranged from a few hours to 16 years [median 2.75 (0.65-6.63)]. PICU LOS ranged from 1 to 56 days [median 6.1 (4-9.9)]. MV duration ranged from 0 to 29.9 days [median $1.2(0.9-5)]$. PRISM score ranged from 0 to 26 [median 6 (3-10)]. Bypass time ranged from 32 to $284 \mathrm{~min}$ [median 109 (77-144)]. Aortic clamping time ranged from 0 to 129 min [median 50 (31-66)]. Mortality was $3.5 \%$.

\section{Persistent LSVC Patients}

Forty-seven children had persistent LSVC (12.7\%), and persistent LSVC was identified on echocardiography before surgery in 39 patients $(83 \%)$; in $5(10 \%)$ patients the innominate vein was present. Thirteen $(28 \%)$ patients presented with a congenital malformation in relation with some kind of obstruction of the left-sided inflow, i.e., interrupted aortic arch, Shone complex, hypoplastic left heart.

In 37 patients $(80 \%)$, persistent LSVC was associated with dilatation of the coronary sinus. Before surgery, echocardiography did not demonstrate any kind of flow acceleration at the inflow of the left ventricle (LV) in this group of patient. In three patients, in whom surgical intervention included closure of an ASD as part of the surgery, significant inflow obstruction of the LV developed after surgery leading to low output syndrome or secondary pulmonary hypertension.

In eight patients (17\%), persistent LSVC was associated with a partially or completely unroofed coronary sinus and in two cases $(4 \%)$ with coronary sinus ostial atresia. Partially unroofed or completely unroofed coronary sinus was only suspected in four patients $(50 \%)$ before surgery and was diagnosed at the time of surgery in two others secondary to unexplained low arterial saturation at the end of bypass. In these cases, if the surgery was a surgical correction with complete partition of the pulmonary and systemic circulation, persistent LSVC was redirected during surgery to the right atrial appendage either directly or with interposition of a Gore-Tex tube graft (W. L. Gore \& Associates, Inc., Flagstaff, Arizona, USA). Coronary sinus ostial atresia was neither diagnosed before surgery nor during surgery but in all cases was diagnosed during the PICU stay using angiography.
PICU Outcome Analysis

Baseline and outcome data are shown in all patients (Table 1), i.e., the persistent LSVC group and the control group (Table 2). Compared with the control group, the persistent LSVC group was significantly younger. There were no statistical differences in bypass time, aortic clamping time, RACHS score repartition, and PRISM score between both groups. Duration of MV was significantly shorter in the control group compared with the persistent LSVC group, whereas PICU LOS did not differ. Bypass time, aortic clamping time, and duration of MV and PICU LOS were not significantly different between patients with persistent LSVC known before surgery and those in whom persistent LSVC was unknown before surgery.

Mortality was also significantly lower in the control group than in the persistent LSVC group. When looking at the causes of death in the patient group with persistent LSVC, they could be directly linked to persistent LSVC in four $(80 \%)$ of five deaths. Two patients had a low cardiac output secondary to acute obstruction of the inflow of the LV with multiple organ failure. Due to coronary sinus ostial atresia, one patient died after Fontan palliation from acute LV dysfunction secondary to venous myocardial infarction. One patient died after intractable chylothorax secondary to complete thrombosis of persistent LSVC that was redirected to the right atrial appendage. Of the five patients who died after surgery, persistent LSVC and its associated anomalies were not detected before surgery in three patients $(60 \%)$.

\section{Univariate Analysis}

Univariate analysis identified other possible factors than persistent LSVC that could be associated with mortality, PICU LOS, or MV duration (Tables 3, 4, 5) as follows: young age $(<1$ year old), bypass time $(>120 \mathrm{~min})$, and PRISM score $(>10)$. RACHS score was not a risk factor for mortality, PICU LOS, or MV duration.

\section{Discussion}

Persistent LSVC may be a benign finding in patients with normal anatomy of the heart. Most of the time, drainage occurs through the coronary sinus to the right atrium without hemodynamic consequences. However, in case of congenital heart malformations (most of the time complex ones), persistent LSVC may be associated with unroofed coronary sinus, partially unroofed coronary sinus, or coronary sinus ostial atresia. All of these associated anomalies could lead to arterial desaturation and paradoxical systemic emboli or to venous myocardial infarction in the case of coronary sinus ostial atresia. 
Table 1 Baseline characteristics of patients

\begin{tabular}{lc}
\hline Characteristics & Patients $(n=371)^{\mathrm{a}}$ \\
\hline Age (year) & $2.75(0.65-6.63)$ \\
Bypass time (min) & $109(77-144)$ \\
Aortic clamping time (min) & $50(31-66)$ \\
No. RACHS 2 (\%) & $186(50.5)$ \\
No. RACHS 3 (\%) & $153(41.6)$ \\
No. RACHS 4 (\%) & $29(7.9)$ \\
PRISM score & $6(3-10)$ \\
PICU LOS (days) & $6.1(4.0-9.9)$ \\
MV duration (days) & $1.2(0.9-5.0)$ \\
No. mortality (\%) & $13(3.5)$ \\
\hline
\end{tabular}

$R A C H S$ risk adjustment for congenital heart surgery, PRISM pediatric risk of mortality, PICU Pediatrics intensive Care Unit, LOS length of stay, $M V$ mechanical ventilation, $L S V C$ left superior vena cava

${ }^{a}$ Data are presented as median and interquartile range or $N(\%)$

In the literature, reports or series of cases describe the potential problems due to persistent LSVC and associated anomalies before, during, and after congenital cardiac surgery [1, 4, 6, 10, 19]. In this context, the results of our study gives some indication of the impact of persistent LSVC during the perioperative period of congenital heart surgery in children. They also suggest that persistent LSVC is associated in this selected population with increased perioperative mortality and MV duration.

Our data showed that persistent LSVC could be missed by echocardiography, and unfortunately there is no clue that could warn about the presence of such. In all cases, the cardiologist should be aware of different signs that may raise the suspicion of persistent LSVC, e.g., unusually low oxygen saturation for a particular congenital heart disease or unusual dilatation of the left atrium in cases of cyanotic cardiac malformation [14, 20].

The review of our data also showed that for congenital heart disease, the associated lesions of persistent LSVC may only be discovered during palliative or corrective surgery when unexplained arterial desaturations,
Table 3 Univariate regression analysis: mortality

\begin{tabular}{lc}
\hline & OR $(95 \% \mathrm{CI})$ \\
\hline Age $<1$ year & $5.0(1.5-16.6)$ \\
Bypass $>120$ min & $9.2(2.0-42.1)$ \\
PRISM score $>10$ & $14.5(3.1-68.3)$ \\
RACHS & $1.7(0.5-5.2)$ \\
Persistent LSVC & $4.7(1.5-15.0)$
\end{tabular}

$R A C H S$ risk adjustment for congenital heart surgery, PRISM pediatric risk of mortality, $L S V C$ left superior vena cava

ventricular dysfunction, or paradoxical emboli occur. These unexpected findings lead to an unexplained complicated postoperative period. If the cause can be identified in the operating room, it is generally necessary to create a second bypass to fix the problem regarding the persistent LSVC. When the cause can not be identified in the operating room, the child is transferred to the PICU, and contrast echocardiography, cardiac catheterization, or other imaging modality is needed to determine the cause of the complication.

Moreover, in a case series by Attenhofer et al. [1] of 25 patients with congenital heart disease and partial unroofed coronary sinus, this diagnosis was missed in 14 patients (56\%) during previous palliative surgery. Filippini et al. [7] reported the problematic association of Glenn and Fontan palliation with associated persistent LSVC. In this case series, persistent LSVC was excluded in all patients either by echocardiography or by catheterization at the time of bidirectional Glenn procedure. However, probably secondary to the high venous pressure system in these patients, the LSVC reopened and lead to arterial desaturation, thus requiring the need for urgent reintervention. Finally, Santoscoy et al. [16] reported five patients with persistent LSVC draining into the coronary sinus and coronary sinus ostial atresia. In these cases as well, the diagnosis was incidental at the time of surgery in three patients. The investigators urged the need to recognize this anomaly before initiating cardiopulmonary bypass to avoid
Table 2 Characteristic of patients in the persistent LSVC group and the control group

$R A C H S$ risk adjustment for congenital heart surgery, PRISM pediatric risk of mortality, $P I C U$ Pediatrics intensive Care Unit, $L O S$ length of stay, $M V$ mechanical ventilation, $L S V C$ left superior vena cava

a Data are presented as median and interquartile range $N(\%)$

\begin{tabular}{lccc}
\hline Characteristics & Persistent LSVC $(n=47)$ & Control $(n=324)$ & $p$ \\
\hline Age (year) & $0.7(0.3-4.5)^{\mathrm{a}}$ & $3.2(0.8-6.9)$ & $<0.001$ \\
Bypass time (min) & $120(85-164)$ & $107(76-143)$ & 0.11 \\
Aortic clamping time (min) & $49(30-73)$ & $51(32-65)$ & 0.83 \\
No. RACHS 2 (\%) & $25(53.2)$ & $161(50.1)$ & \\
No. RACHS 3 (\%) & $18(38.3)$ & $135(42.1)$ & 0.89 \\
No. RACHS 4 (\%) & $4(8.5)$ & $25(7.8)$ & 0.11 \\
PRISM score & $8(4-12)$ & $6(3-10)$ & 0.53 \\
PICU LOS (days) & $6(4-14)$ & $6(4-9)$ & 0.04 \\
MV duration (days) & $3.0(1.0-6.0)$ & $8.2(0.8-4.7)$ & 0.004 \\
No. mortality (\%) & $5(10.6)$ & $8(2.5)$ & \\
\hline
\end{tabular}


Table 4 Univariate regression analysis: mechanical ventilation duration

\begin{tabular}{lc}
\hline & OR $(95 \% \mathrm{CI})$ \\
\hline Age $<1$ year & $12.8(5.7-28.6)$ \\
Bypass $>120 \mathrm{~min}$ & $1.7(1.1-2.7)$ \\
PRISM score $>10$ & $3.2(1.7-5.8)$ \\
RACHS & $1.1(0.7-1.7)$ \\
Persistent LSVC & $2.5(1.1-5.6)$ \\
\hline
\end{tabular}

$R A C H S$ risk adjustment for congenital heart surgery, PRISM pediatric risk of mortality, $L S V C$ left superior vena cava

Table 5 Univariate regression analysis: PICU LOS

\begin{tabular}{ll}
\hline & OR $(95 \% \mathrm{CI})$ \\
\hline Age $<1$ year & $3.8(2.4-6.1)$ \\
Bypass $>120 \mathrm{~min}$ & $1.9(1.2-2.9)$ \\
PRISM score $>10$ & $2.7(1.6-4.3)$ \\
RACHS & $1.4(0.9-2.1)$ \\
Persistent LSVC & $1.4(1.0-2.5)$ \\
\hline
\end{tabular}

RACHS risk adjustment for congenital heart surgery, PRISM pediatric risk of mortality, $L S V C$ left superior vena cava

disastrous consequences secondary to obstruction of the LSVC. The challenge, then, is to identify these associated lesions before surgery for complex or simple congenital malformations, such as single ventricle, septal shunts, extracardiac shunts, etc.

In our retrospective review, we encountered all of the issues described in the literature related to persistent LSVC, e.g., obstruction of the mitral inflow and consequently low cardiac output and pulmonary hypertension, coronary sinus ostial atresia and ventricular dysfunction on increased coronary venous pressure, etc. All of these complications may explain the increased mortality and the prolonged MV time in patients with persistent LSVC. If the lesions are promptly identified and surgery is performed consequently, most of these problems can be solved and therefore lead to no other complication. In our center, every patient with a diagnosis of persistent LSVC is closely evaluated by echocardiography, and, when necessary, contrast echocardiography is performed with injection from a peripheral vein of the left arm as advocated by many investigators [20]. If a doubt persists, angiography is performed.

Our study has limitations. The retrospective nature of this cohort of surgical patients may introduce some bias into analyses of the data. In contrast, it would be complex to initiate a prospective study on persistent LSVC if this is associated with a greater risk for the patient. The cohort is described over a period of many years, and some surgical techniques may have evolved and thus may contribute to changes in postoperative outcomes. Nevertheless, to our knowledge, this is the largest series of surgical cases with persistent LSVC.

\section{Conclusion}

Persistent LSVC is not an uncommon finding in patients with congenital heart disease. Most often it does not affect cardiac surgery or postoperative outcomes. In cases of complex congenital lesions, persistent LSVC may be associated with anomalies of the inflow of the systemic ventricle, unroofed coronary sinus, partially unroofed coronary sinus, and coronary sinus ostial atresia. These associations can be masked at the time of preoperative evaluation and revealed only incidentally at surgery or after surgery, thus leading to unexpected complications, such as arterial desaturation, paradoxical emboli, or ventricular dysfunction.

We recommend comprehensive preoperative imaging in all patients in whom persistent LSVC is recognized. Once the exact diagnosis is made, the surgeon can fix the issue at the time of surgery.

Acknowledgments Thanks to Jean-Michel Manzano and Fabrice Weyermann (PICUs, University Hospital Center and University of Lausanne, Switzerland) for informatics support.

Conflict of interest The authors declare that they have no conflict of interest.

\section{References}

1. Attenhofer Jost CH, Connolly HM, Danielson GK, Dearani JA, Warnes CA, Jamil et al (2007) Clinical features and surgical outcome in 25 patients with fenestrations of the coronary sinus. Cardiol Young 17:592-600

2. Awasthy N, Ambatkar P, Radhakrishnan S, Iyer KS (2012) Lutembacher syndrome with unroofed left superior vena cava: a diagnostic dilemma. Pediatr Cardiol

3. Bonardi M, Valentini A, Camporotondo R (2012) Unroofed coronary sinus and persistent left superior vena cava: a case report. J Ultrasound 15:179-182

4. Buyuklu M, Ufacik O, Pirhan O, Tatli E (2009) A rare association recognized before cardiac surgery: persistent left superior vena cava and secundum atrial septal defect. Int $\mathrm{J}$ Cardiol 132:e82-e84

5. Cherian SB, Ramesh BR, Madhyastha S (2006) Persistent left superior vena cava. Clin Anat 19:561-565

6. Erdogan M, Karakas P, Uygur F, Mese B, Yamak B, Bozkir MG (2007) Persistent left superior vena cava: the anatomical and surgical importance. West Indian Med J 56:72-76

7. Filippini LH, Ovaert C, Nykanen DG, Freedom RM (1998) Reopening of persistent left superior caval vein after bidirectional cavopulmonary connections. Heart 79:509-512

8. Heye T, Wengenroth M, Schipp A, Johannes Dengler T, Grenacher L, Werner Kauffmann G (2007) Persistent left superior vena cava with absent right superior vena cava: morphological 
CT features and clinical implications. Int J Cardiol 116:e103e105

9. Larsen SH, Pedersen J, Jacobsen J, Johnsen SP, Hansen OK, Hjortdal V (2005) The RACHS-1 risk categories reflect mortality and length of stay in a Danish population of children operated for congenital heart disease. Eur J Cardiothorac Surg 28:877-881

10. Neema PK, Manikandan S, Rathod RC (2007) Absent right superior vena cava and persistent left superior vena cava: the perioperative implications. Anesth Analg 105:40-42

11. Pollack MM, Ruttimann UE, Getson PR (1988) Pediatric risk of mortality (PRISM) score. Crit Care Med 16:1110-1116

12. Povoski SP, Khabiri H (2011) Persistent left superior vena cava: review of the literature, clinical implications, and relevance of alterations in thoracic central venous anatomy as pertaining to the general principles of central venous access device placement and venography in cancer patients. World J Surg Oncol 9:173

13. Ramos N, Fernandez-Pineda L, Tamariz-Martel A, Villagra F, Egurbide N, Maitre MJ (2005) Absent right superior vena cava with left superior vena cava draining to an unroofed coronary sinus. Rev Esp Cardiol 58(8):984-987

14. Rigatelli G (2007) Congenitally persistent left superior vena cava: a possible unpleasant problem during invasive procedures. J Cardiovasc Med (Hagerstown) 8:483-487

15. Rogers K, Bader F, Gilbert E (2009) Performing a myocardial biopsy in a cardiac transplant patient with a persistent left superior vena cava. Cathet Cardiovasc Interv 74:295-297
16. Santoscoy R, Walters HL 3rd, Ross RD, Lyons JM, Hakimi M (1996) Coronary sinus ostial atresia with persistent left superior vena cava. Ann Thorac Surg 61:879-882

17. Schreve-Steensma AM, van der Valk PH, Ten Kate JB, Kofflard MJ (2008) Discovery of a persistent left superior vena cava during pacemaker implantation. Neth Heart J 16:272-274

18. Simsic JM, Cuadrado A, Kirshbom PM, Kanter KR (2006) Risk adjustment for congenital heart surgery (RACHS): is it useful in a single-center series of newborns as a predictor of outcome in a high-risk population? Congenit Heart Dis 1:148-151

19. Taweevisit M, Thorner PS (2011) Persistent left superior vena cava: does it have a role in the pathogenesis of hypoplastic left heart syndrome? Pediatr Dev Pathol 14:105-110

20. Thaiyananthan NN, Jacono FJ 3rd, Patel SR, Kern JA, Stoller JK (2009) Right-to-left anatomic shunt associated with a persistent left superior vena cava: the importance of injection site in demonstrating the shunt. Chest 136:617-620

21. Thal S, Boyella R, Arsanjani R, Thai H, Juneman E, Movahed MR et al (2012) Unusual combination of Holt-Oram syndrome and persistent left superior vena cava. Congenit Heart Dis 7: E46-E49

22. Wissner E, Tilz R, Konstantinidou M, Metzner A, Schmidt B, Chun KR et al (2010) Catheter ablation of atrial fibrillation in patients with persistent left superior vena cava is associated with major intraprocedural complications. Heart Rhythm 7:1755-1760 